\title{
Understanding Networked Collaboration: Fields and Patches of Interactions.
}

\author{
Lilian Henze ${ }^{1}$, Ingrid Mulder ${ }^{1,2}$, Pieter Jan Stappers ${ }^{1}$ \\ ${ }^{I}$ ID-StudioLab, Delft University of Technology, Landbergstraat 15, 2628 CE Delft, the Netherlands \\ \{l.a.r.henze, i.j.mulder, p.j.stappers\}@tudelft.nl \\ ${ }^{2}$ Creating 010, Rotterdam University, Wijnhaven 99, 3011 WN Rotterdam, The Netherlands
}

\begin{abstract}
The increased complexity in Product Service Systems development goes hand in hand with complex heterogeneous networks. A Human-Centred Design approach has been taken to understand such networked collaboration and to develop a framework having user-experiences guiding communication and collaboration among parties. The current work elaborates on the development of a framework for networked collaboration inspired by literature research of Actor Network Theory and Boundary Objects as well as insight gained in case studies and workshops. Iterative design of the framework has led to a 'landscape of interactions'. This landscape is applied as means to understand the networked collaborations and the designating of tools for designers in Product-Service System development.
\end{abstract}

\section{Keywords}

Product Service Networks, networked collaboration, ANT, boundaries, boundary objects, human-centred design.

\section{Introduction}

I found it difficult with Product-Service System development to develop a vision. And once we had that, to roll out the vision in the creation and realization phase. It is more difficult because of the increasing complexity of the networks involved'.

'Managers still work in a system that doesn't cope with network complexity'.

'It is the complexity of the networks, there are more values involved you need to take into consideration'.

These quotes are from a conversation between a product-service developer and an innovation consultant discussing the changes they faced in their professional practice. The discussion happened during a recently held workshop, one of a series in a research project aiming at developing a framework of methods, techniques and tools enabling the conceptualization and communication of product-service systems (PSSs) [Henze, Mulder \& Stappers, 2011]. The present research project studies PSS development from a Human-Centred Design (HCD) perspective; meaning that the user's experiences guide all parties involved in the networked collaborations [Stickdorn and Schneider, 2010]. These parties concern e.g. designers, researchers, business developers, business managers, operators and customers all coping with a rapid changing world.

The opening quotes nicely address the need for developing such a framework of methods, techniques and tools: the increasingly complexity of the development of product-service systems (PSSs) in heterogeneous networks of Small and Medium Enterprises (SMEs) and departments in larger organizations with some kind of end-user involvement. Not only are methods, techniques and tools that support understanding end-users and deploy this understanding in the PSS development are needed [Henze, Mulder \& Stappers, 2011] but also an understanding of the needs of the collaborating parties is necessary in order to apply the relevant methods, techniques, and tools. For the purpose of this paper we use the term 'tools' for methods, techniques and tools. 
According to Manzini et al. 2001, industries look for products and services jointly capable of fulfilling clients' needs with higher added value and a smaller environmental impact as compared to an existing product or system. PSS design is about creating value for both clients and organizations [Henze et al. 2012]

The increased complexity the discussants are referring to seem to be the result of the emerging heterogeneous networks involved and at the same time getting the Human-Centred Design (HCD) process aboard in their organizations. Dealing with the complexity and imbedding the HCD process lead to a coherent ecosystem flexible enough to enable innovation in a continuous changing environment.

The current work reports on the development of a framework that enables understanding networked collaborations and designating the tools that support HCD. In an iterative process an initial framework is redesigned into a landscape of fields of interactions between actors and objects involved. It covers the PSS development process, starting from understanding user needs through generating product service propositions and developing these into implementations. We discuss how the framework can be used to describe collaborations and how to select the tools that optimize these collaborations in the remainder of this work.

\section{A pragmatic approach}

The current study has taken a pragmatic approach as both theory and practice regarding PSS development are discussed in regular workshops of the research team in which the proposed framework was collaboratively constructed (figure 1).

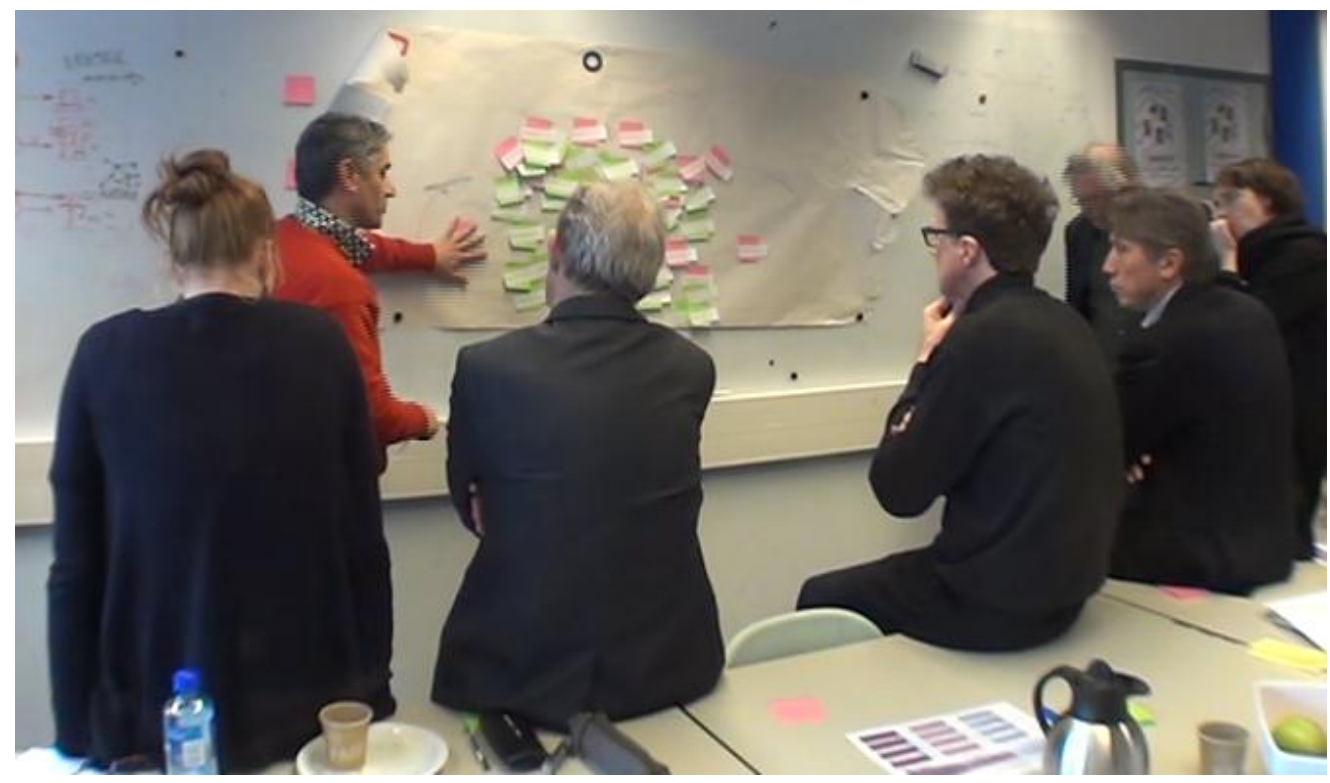

Figure 1: academic and industry partners are discussing the application of the initial framework during a recent project workshop.

The research team includes academic and industrial professionals from various disciplinary backgrounds among other industrial design, change management, software and service engineering, HCD, organisational development, business strategy, service design having about 15-25 years of project experiences regarding PSS and/or networked collaboration. Case studies are conducted with the industrial partners, each case with a focus on different aspects of PSS development. During each case several iterations are made of design and evaluation leading to improvements in the PSS concept, improvements in the PSS design method, and improvements of the framework. The case studies are presented in the project workshops. Next to these project workshops and cases we collaborated in an academic team on the topic of boundary objects. By 
actually designing boundary objects for a scenario, from the real practice in NPD, we gained insights on the boundary objects and the boundaries they are bridging [Houssian et al. 2012].

All workshops were videotaped and analysed to get a deeper understanding of the collaborations in PSS development and the related boundaries and boundary objects.

\section{From actors to interactions}

In earlier work [Henze, Mulder \& Stappers, 2011] we defined an initial framework to represent all parties collaborating in the PSS development. In particular, we distinguish between networks of service design, organizations, and networks of people experiencing the products and/or services.

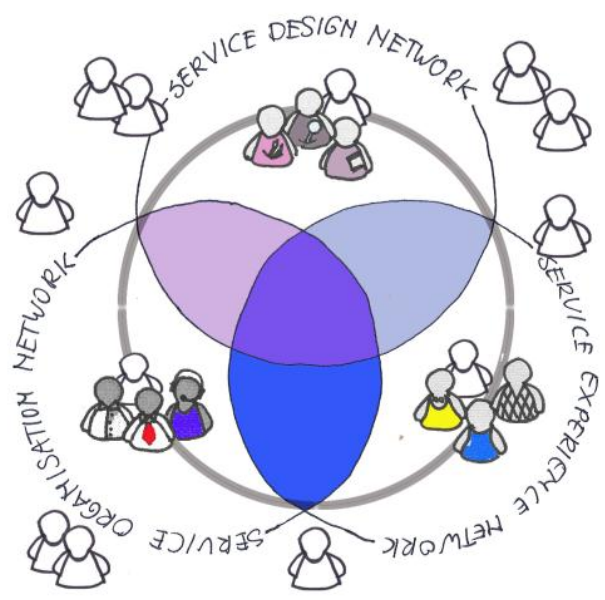

Figure 2: The initial framework stresses the interactions on the boundaries of the networks, which separate the actors, and form barriers in connections across boundaries, but the connections remain implicit.

Although this initial framework helped to bring out the complexity it does not explain the difficulties in collaborations. It only represents the 'actors' and does not elaborate on the actors' connections or interactions. It only shows networks with boundaries without making the connections (collaborations) explicit. Where networks can be defined as 'general yet powerful means of representing patterns of connections or interactions between parts of a complex system' [Newman, 2010], the initial framework fails this representation. A deeper understanding of the PSS is sought to include a more explicit representation of the interactions in the framework. These interactions do not only take place between social networks (a representation of actors and ties), but also technological networks (physical networks of components) and information networks (linked databases). For instance one of the cases in the current project shows the complexity of PSS development. Because the development teams, in this case, use the agile method Scrum, where self-organizing teams focus on rapid iterations with continuous customer input along the way [Deemer et al. 2010], there is a need to inform all stakeholders involved on constant change. The software development teams (with different workplaces located in different countries) share information with colleagues from different departments during teleconference meetings (in a meeting room with videoconference technology) and via intranet (email, twitter, blogs and databases). Informal exchange of experiences and information takes place in hallway meetings (e.g. during gatherings in front of the coffee machines).

The example shows that a mix of all the networks (social, technological and information) influences the interactions. This is in keeping with ANT [Latour, 2005]: both humans and nonhumans (Latour uses the word 'actants' for humans and non-humans) have agency, they form the vertices in the network (figure 3) while the interactions/connections form the edges. 


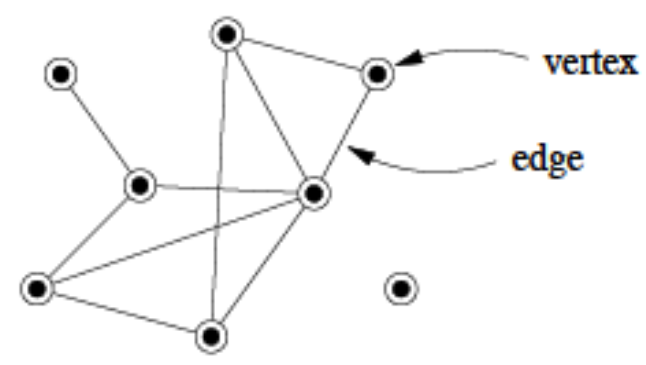

Figure 3: a simple network (from Newman, 2011), the actants form the vertices and the interactions/connections form the edges.

Such a network represents an action in accomplishing translations and transformations [Carlile, 2004]. The representation of the PSS development in a network of actants and interactions will make it possible to trace all activities that lead to translations and transformations and thus understand the networked collaborations. However representing all actants involved as a vertex and their individual interactions/connections as edges would lead to a complex and saturated picture where structure is hard to find. In the following section a simplification of this network is proposed.

\section{Fields and patches of interactions}

Interestingly, results showed that the tools we discussed in the projects workshops were too general to designate to a specific interaction in PSS development. In one of the workshops we used an overview of tools, represented in a matrix that was based on the analyses of case studies and discussions between research team members, to decide on case studies we wanted to do in the remainder of the research project (figure 4).

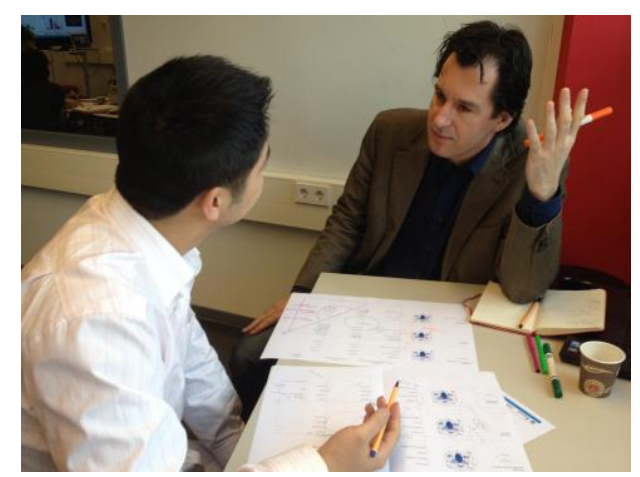

Figure 4: two workshop participants using the matrix with tools in discussing the aspects they wanted to focus on in a future case study.

In this matrix the development phases labelled as explore, vision and create were combined with the strategic (the PSS), tactic (the concept propositions) and operational (the products/services) level of developments. The matrix was intended to assign tools for exploration (e.g. interviews, observations), mapping (e.g. stakeholder mapping), sharing (e.g. video, visualizations) and evaluating (e.g. workshops, usability testing). The initial framework (figure 2) was integrated in the matrix to indicate 'who was involved'.

All participants faced difficulties in using the matrix. One of the participants expressed this by saying: 'For me this is all a bit arbitrary, we are throwing away all information we already have learned in the retrospective cases and which is applicable in practice'. 
The initial framework didn't bring us a better understanding of the networked collaboration and the barriers and enablers in PSS development. It didn't show the interactions and thus could not help us to trace the activities that lead to translations and transformations. In a following workshop (see figure 1) we returned to the initial framework and found both tools descriptions and visualization of the framework lacked detail to get an overview of interactions and all relevant tools. The 'one big cloud' of tools (the post its in the middle of the poster) in figure 1 illustrates this lack of overview.

In the following section we elaborate on the redesign of the framework into a landscape of fields of interactions.

\subsection{A Landscape of Fields and Patches}

As mentioned in section 3 for the PSS some grouping of actants and interactions, in a way that the complexity of PSS is still acknowledged, is needed. The grouping of similarities in interactions in PSS development leads to patches of activities. Each patch represents networks (as a representation of an activity) accomplishing translations and transformations. These activities concern practices of what people do, how they do it and why they do it [Levina and Vaast, 2005]. Patches are grouped into a field of interactions, each field representing the activities in the 'networks' of the initial framework: design, organization and experience. Taking the initial framework a step forward with this in mind, it is now visualized as a landscape of fields of interactions (figure 5).

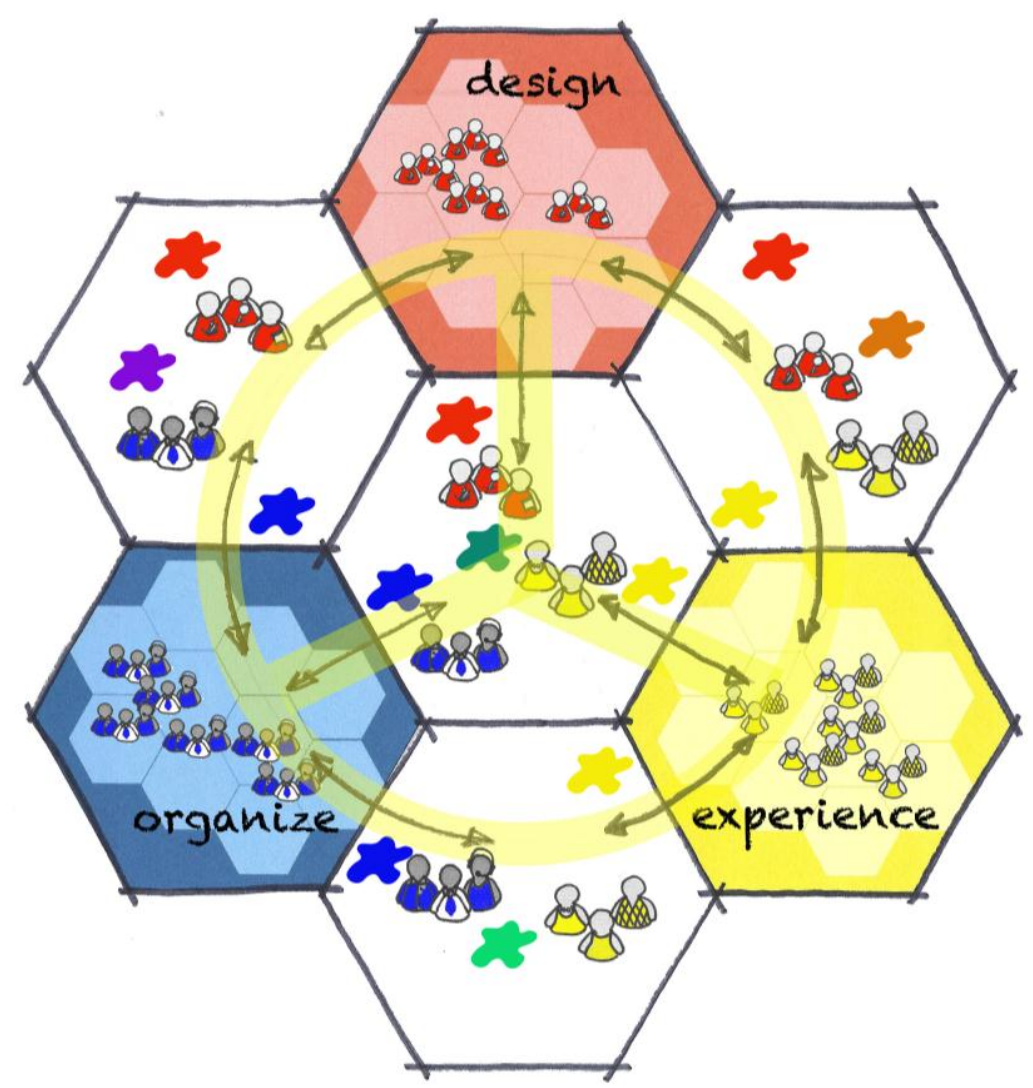

Figure 5: Landscape of activity fields divided into fields of design activities (red), organization activities (blue) and experiences (yellow). The white fields give room for heterogeneous interactions aiming at collaborations of designers (red puppets), organizers (blue puppets) and experiencers (yellow puppets) in PSS development. The yellow circle visualizes the user experiences guiding all interactions.

In the HCD approach the experiences of everyday life (the yellow Experience field) are guiding all interactions in the landscape. The Design field (the red field) translates and transforms these experiences into PSS concepts that are translated and transformed into PSS propositions by the Organize field (the blue field). The propositions are delivered to the Experience field, where the 
products and services are used thus facilitating (and influencing) the everyday life experiences. The yellow circle in figure 5 visualizes this process. The double-sided arrows indicate this is not a one-way, serial, process; it is a continuous process consisting of local research, creation and evaluation activities. The smaller patches in the design, organize and experience fields visualize the groups of practice in the different fields. All practices differ in the networks of people and objects (e.g. tools, procedures, interests, values) accomplishing translations and transformations.

The design field is formed by patches representing design practices, such as among others service design, architecture, software development, business consultancy and industrial design.

In the organize field the patches represent practices as marketing, support, sales, business management, finance. Patches representing practices of use form the experience field; these depend on the specific PSS development. The white fields are formed by activities as research, co-creation, advising and communication.

One of the cases was about the development of a remote health care PSS. Service design collaborated with project management 'remote care' and elderly clients in a research to understand the needs of the clients (the users of the remote care); interactions from the middle white field. Service design collaborated with a new media designer in translating the insights into PSS concepts, interactions in the red design field. Business consultancy interacted with the designers to translate the concepts (visualized in blue prints) into a social business case, the social business case report should serve to bring the organize field into action. But as one of the project managers said: 'to say it very bluntly, we created very nice concepts but the organization just didn't understand what we were talking about, we had very nice concepts and booklets explaining these concepts, but they just didn't get it'. Obviously the interactions did not lead to transformation of the concepts into propositions. Tracing back the interactions learn that in this case not all relevant patches in the organize field were connected. Some patches, e.g. the municipality government patch and the infrastructure patch (in this case an internet provider) were not adequately connected.

\subsection{Interactions, Boundaries and Boundary Objects}

The example in the previous section shows that in the PSS development translations and transformations are not always accomplished: boundaries occur. Boundaries are defined as the differences that lead to discontinuity in (inter) action [Akkerman and Bakker, 2011]. These boundaries can be crossed by means of boundary spanners (an individual crossing boundaries) and boundary objects: objects which are both plastic enough to adapt to local needs and constraints of the parties employing them, yet robust enough to maintain a common identity across sites [Star and Griesemer, 1989]. For instance in new product development boundary objects (e.g. prototypes, material samples, posters, CAD databases) are cornerstones to support development teams consisting of software developers, hardware engineers, interaction designers and usability specialists to work together [Stompff, 2012]. Our hypothesis is that a substantial part of tools that enable PSS development can be seen as boundary objects. In keeping with Star, 1989: the creation and management of boundary objects is key in developing and maintaining coherence across intersecting (social) worlds.

In two workshops [Houssian et al., 2012] we explored the possibility to actually create methods and tools that serve as boundary objects. Based on a scenario, from the real practice in NPD, we explored boundaries, and designed tools to solve the boundary issues we framed. As a result we created a toolkit of boundary objects and interventions. A striking observation in these workshops was that we only could create boundary objects when provided with a clear understanding of the boundaries we are facing. This understanding proved to be difficult in the workshops, thus confirming the necessity of a framework supporting this understanding. 


\subsection{Designating tools}

To apply the framework for understanding the boundaries and designating for PPS development relevant tools each field (and/or patch) can be isolated and used as a canvas for describing interactions, trace boundaries and grouping the tools supporting these interactions.

As an example, the canvas in figure 6 contains design research tools enabling interactions between design researchers and users (who experience the PSS in their everyday life).

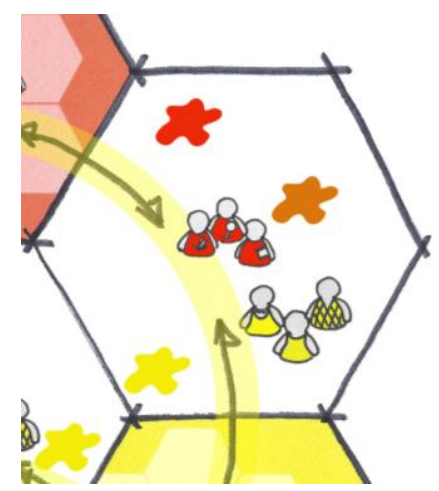

Figure 6: One of the fields of the landscape serving as a canvas for understanding boundaries and designating tools (described on the red, orange and yellow spots) supporting design research.

The red tools focus on supporting designers in communicating the results of the research to others interacting in the design field to deploy actual experiences of people as drivers for PSS design. Examples of the red tools are personas, experiencing prototypes etc.

The orange tools focus on supporting designers and experiencers to exchange experiences, e.g. generative tools. The yellow tools support the experiencers in communicating their experiences e.g. probes and diaries.

Reassembling the filled canvasses of patches and fields results in a landscape of tools, representing all relevant tools for PSS development: the networked collaboration canvas.

\section{Discussion and Conclusions}

The redesign of an initial framework of methods, techniques and tools that enables the conceptualization and communication of product-service systems, resulted in a landscape of fields and patches of interactions. The redesign was necessary to get a grip on the networked nature of the collaborations within a HCD approach in PSS development. The landscape was built on the notions from ANT and Boundary Objects and focuses on interactions between actors and objects involved. It covers the full life cycle of a PSS development process, starting from understanding user needs through generating product service propositions and developing these into implementations. The process is an accumulation of translations and transformations. The new framework, the networked collaboration canvas, is applied twofold: a) as a means to understand the networked collaborations and b) as a means to designating of tools that support PSS development.

In recent case studies (see figure 7) the landscape is used to reflect on projects and identify boundaries and possible improvements (and find tools to do so) such as: do not only focus on identification of user needs but also identify organization needs, prototype the PSS concepts in appropriate detail for evaluation and communication. 


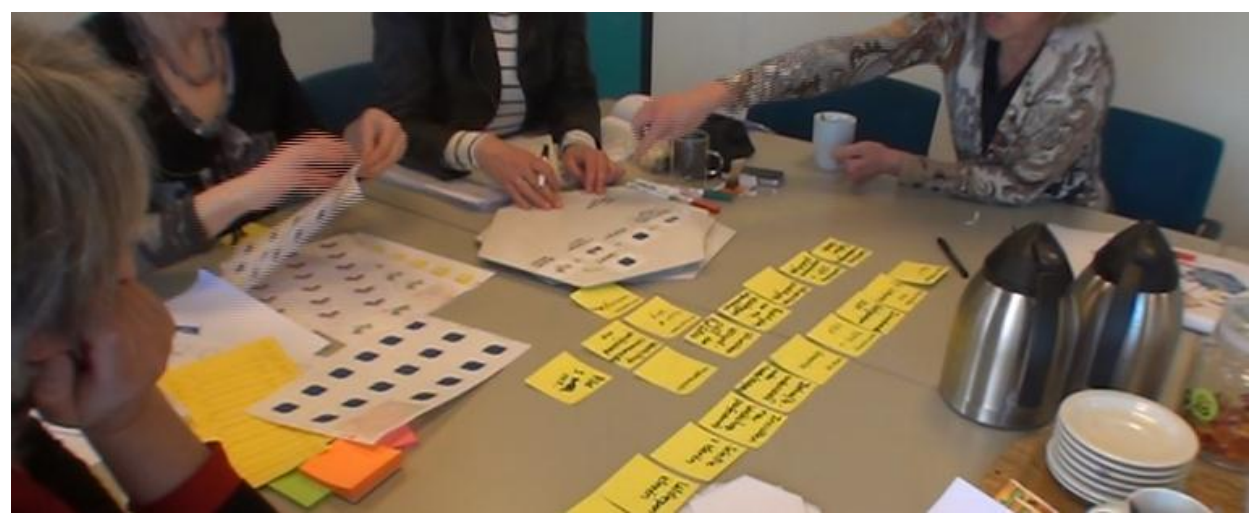

Figure 7: Using the canvas

The framework needs further validation, this will be done based on future case studies and workshops.

The focus in this research project is on the role of design in PSS development, but the cases and workshops show that the landscape could also serve the field of organization and technology management in understanding PSS development and collaboration with design.

\section{Acknowledgement}

This work is part of the 'Creative Industry Scientific Programme' (CRISP), supported by the Dutch Ministry of OCW. We thank all partners involved in the CRISP PSS 101 project; industrial partners are Four C management, STBY, Exact, Océ industries, and Zuidzorg. Academic partners are from the Design Academy Eindhoven as well as the Delft University of Technology, both the Faculty of Industrial Design Engineering and the Faculty of Technology, Policy and Management. We wish to acknowledge our gratitude and appreciation to Aaron Houssian (Delft University of Technology), Malcolm Jones, Vicky Teinaki and Michael Leitner (all Northumbria University), with whom we spend four workshop days exchanging knowledge and exploring together the ins and outs of boundary objects.

\section{References}

Akkerman, S. F. \& Bakker, A. 2011. Boundary crossing and boundary objects. Review of Educational Research, 81, 132-169.

Carlile, P. R. 2004. Transferring, translating, and transforming: An integrative framework for managing knowledge across boundaries. Organization science, 555-568.

Deemer, P., Benefield, G., Larman, C. \& Vodde, B. 2010. The Scrum Primer. Scrum Primer is an in-depth introduction to the theory and practice of Scrum, albeit primarily from a software development perspective, available at: http://assets.scrumtraininginstitute. com/downloads/1/scrumprimer121. pdf, 1285931497.

Henze, L., Mulder, I., Stappers, P.J. 2011. Conceptualizing Product Service Networks: Towards an Initial Framework. International Conference on Concurrent Enterprising. Aachen.

Henze, L., Mulder, I., Stappers, P.J., Rezaei, B. 2012. Right Service \& Service Right, how collaborating heterogeneous networks at the front end of service development benefit the process to get the service right. ServDes.2012, co-creating services. Service Design and Innovation Conference. Helsinki, Finland, 2012.

Houssian, A, Mulder, I., Henze, L., Jones, M., Teinaki, V., Leitner, M. 2012. Crossing Boundaries: Exploring What design Can Do, available at: http://boundariescrossing.wordpress.com/topic/.

Latour, B. 2005. Reassembling the social: An introduction to actor-network-theory, Oxford University Press, USA.

Levina, N. \& Vaast, E. 2005. The emergence of boundary spanning competence in practice: implications for information systems' implementation and use. MIS Quarterly, 29, 335-363.

Manzini, E., Vezzoli, C. \& Clark, G. 2001. Product-service systems: using an existing concept as a new approach to sustainability. Journal of Design Research, 1, 12-18.

Newman, M. 2010. Networks: an introduction, Oxford University Press, Inc.

Star, S. L. \& Griesemer, J. R. 1989. Institutional ecology,translations' and boundary objects: Amateurs and professionals in Berkeley's Museum of Vertebrate Zoology, 1907-39. Social studies of science, 19, 387.

Stickdorn, M. \& Schneider, J. 2010. This is Service Design Thinking. Amsterdam: BIS Publishers.

Stompff, G. 2012. Facilitating Team Cognition: How designers mirror what NPD teams do. doctoral thesis, Delft University of Technology. 